\title{
Algunas Observaciones sobre Tolserol en el Parto
}

\author{
Doctor Efraín González
}

Médico del Departamento de Obstetricia del Hospital de San José de Bogotá.

\section{3-0 - TOLOXI I-2 PROPANODIOL}

Sinónimos: Mỵanesin, Mefanesín. Tolserol.

Fue sintetizado en Inglaterra en 1946 por Berger y Brandley e inicialmente hicieron sus estudios experimentales en animales para averiguar sus propiedades larmacológicas y comprobaron que con dosis perfectamente inocuas se producía relajación muscular, lo cual indujo para que se envara en los estados caracterizados por espasmos, contractura, que denotan una hiperactividad muscular.

Otros investigadores concluveron que el sitio de acción del Myanesín se verifica en la célula de conexion neuro-muscular ejerciendo una disminución de climinando el estímulo nervioso que llega a las células de las astas anteriores de la medula espinal desde los centros nerriosos, reduciendo así, el nivel de actividad muscular.

Hay comunicaciones cientificas en las cuales se dice que el Myanesin produce sueño o un estado de somnolencia por su acción selectiva sobre el cerebro, tálamo. Jacobson aconseja esta droga en los estados de ansiedad por su efecto sedante.

\section{Fardiacologia:}

La fórmula química de constitución es la siguiente:

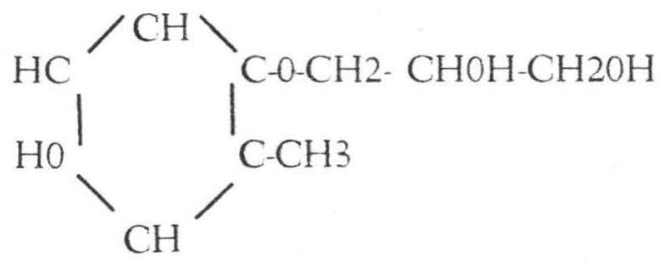

El 3-0 Toloxi - 1-2 Propanodiol, es un compuesto incoloro, inodoro, cristalino, fundible a los 70 y 71 grados C., soluble en agua a 22 grados C. 
Pueden obtenerse soluciones relativamente estables enfriando las soluciones preparadas a más altas temperaturas. Es muy soluble en el alcohol etílico y en el propileno glicol.

ABSORCIÓN, ELIMINACión y REPARACION EN EL ORGANISMO

Se absorbe correstamente por la sangre, músculos, superficies subcutáneas, cavidad peritoneal, recto y estómago.

La repartición en el organismo se hace especialmente sobre el tejido nervioso y el páncreas.

La eliminación se verifica cuando se aplica por vía endovenosa rápidamente $y$ se hace en su totalidad por el riñon.

\section{ACCIÓN SObRe EL aparato GaSTROINTESTINAL:}

Cuando la droga es usada durante largo tiempo por vía oral se pueden producir náuseas y vómitos.

\section{Acción sobre el aparato circulatorio:}

Puede producir taquicardia pasajera. Produce una baja en la tensión arterial especialmente en la minima.

Sobre la sangre, in vitro, se observa hemolisis. En preparaciones de glóbulos rojos con solución salina y tratados luégo con Myanesín en solución 1.32 ocurrió hemolisis en un $25 \%$ en las 24 horas. In vitro, se demostró la hemolisis de la siguiente manera: se invectó 10 c.c. de la solución de Myanesín al 10\% vía endovenosa, luégo se tomaron muestras de sangre a los 45 segundos y a los 85 segundos después de aplicada. Tres minutos más tarde se invectaron 15 c.c. de la solución al $10 \%$ y se tomó muestras de sangre a los 5 minutos después de administrada. Todas las muestras presentaron hemolisis, especialmente la tercera en la cual había un $25 \%$.

La precipitación de las proteínas ocurrió en la sangre humana en soluciones al 1.4 de Myanesín.

\section{Acción sobre el sistema nervioso:}

Se han hecho estudios sobre la influencia que puede tener el Myanesín sobre el electroencefalograma; con aplicaciones rápidas de Myanesín, 50 c.c. por vía endovenosa, las oscilaciones rápidas y de bajo voltaje fueron cambiadas por ondas más lentas y de mavor amplitud.

No produjo cambios definitivos en las descargas producidas por el tálamo, pero el hipotálamo demostró una depresión definitiva, interrumpida por la aparición de ondas de alta amplitud.

En los animales de experimentacién, la inyección intraespinal produce una disminución del tono del cuadriceps seguida de abolición de los reflejos. 
Inhibe la acción del sulfato de estricnina sobre los reflejos, los cuales reaparecen parcialmente comprobando así su acción sobre las células de conexión neuromuscular.

En gatos, con los cordones de la medula espinal aislados, el Myanesín abolió los movimientos de los miembros inferiores, al estimular los nervios periféricos, desapareció el clunus y se redujo su amplitud a un nivel normal.

\section{ACCión SOBRe el Sistema muscular:}

Tiene una acción relajadora sobre los músculos. En animales de experimentación y en pacientes en los cuales se ha usado la droga se ha puesto de manifiesto dicha acción debida a una inhibición de los reflejos de la medula espinal.

\section{ACCIÓN SOBRE EL RIÑón:}

En los exámenes de laboratorio practicados en animales de experimentación $y$ en pacientes tratados con esta droga, el examen de orina presenta hemoglobinuria, o los productos de la desintegración de los glóbulos rojos.

\section{ACCIÓN SOBRE LOS MOVIMIENTOS OCULARES:}

En pacientes que presentaban enfermedades cerebrales $y$ que padecian de nistagmus espontáneo se les aplicé de 30 a 50 c.c. de solución al $10 \%$ de Myanesin por vía endovenosa, desapareciendo el nistagmus. En cambio, en pacientes que no presentaban nistagmus la inyección de Myanesín rápida produjo nistag. mus horizontal.

\section{Dosis:}

Se ha administrado como dosis máxima por vía oral 15 gramos en las 24 hotas, y por vía endovenosa 150 c.c. de la solución al $2 \%$.

\section{OBSERVACIONES HECHAS DURANTE LA APLICACION DEL TOLSEROL EN EL TRABAJO DEL PARTO}

En este estudio se emplea el Tolserol vía endovenosa en la conducción del trabajo del parto comprobando su acción como hipno-analgésico.

Se utilizan soluciones al $1 \%$ preparadas en dextrosa al $5 \%$ en solucion salina o en agua destilada y luégo administrada vía intravenosa gota a gota.

El Tolserol usado en esta forma tiene propiedades sedantes sobre el sistema ervioso central ya que durante las primeras etapas de su aplicación produce ueño tranquilo en las pacientes y analgesia en los momentos en que se presentun las contracciones uterinas sin que altere el ritmo e intensidad de la marcha del trabajo del parto, analgesia que va aumentando a medida que progresa la 
inyección de esta droga, hasta el punto de que las pacientes duermen tranqui lamente al cabo de pocos minutos y sólo se quejan ligeramente con cada contratracción. En los músculos produce una relajacion manifiesta sobre todo en los perineales haciendo por lo tanto que se acorte el periodo de expulsion.

La acción más importante del Tolserol se efectúa sobre el cuello uterino haciendo que el período de dilatación sea más rápido y por lo tanto acorta de esta manera la duración del trabajo del parto. El Tolserol hace que con un trabajo regular y con contracciones uterinas de buena intensidad en un tiempo de una hora o menos se produce un aumento de la dilatacićn en el cuello del útero de 7 u 8 centímetros. Los casos que presentaron trabajos prolongados y distocia de cuellos (Síndrome de Schikelée) el Tolserol venció la distocia y produjo dilatación rápida del cuello.

A medida que se va invectando la droga la paciente experimenta una sensación de vértigo, luégo somnolencia, relajación muscular y más tarde se queda profundamente dormida, a veces puede presentar nistagmus, movimiento incoordinado de los miembros y excitación con euforia.

Como manifestaciones de intolerancia de la droga se presentaron náuseas, vómitos, taquicardia y taquipnea, ligeras modificaciones en la tensićn arterial y que casi siempre son temporales. Por los examenes de laboratorio se pudo demostrar que la inyección intravenosa de Tolserol produce una disminución en el número de hematíes, de la hemoglobina, del hematocrito y del promedio de hemoglobina corpuscular. En cambio, los leucocitos aumentan considerablemente $\mathrm{y}$ en un caso se encontraron granulaciones tóxico-degenerativas en los polinucleares neutrófilos. En los exámenes de orina se hallaron hematies, hemoglobi na, pigmentos biliares, elementos que no existían antes de la aplicación del Tolserol.

La administracićn del Tolserol vía endorenosa está contraindicada en pacientes anémicos o que presenten insuficiencia hepática o renal.

El Tolserol no ejerce accion nociva alguna sobre el feto, va que todos respiran espontáneamente después de la expulsion y su desarrollo ulterior se hace normalmente. No altera la evolución del alumbramiento ni del puerperio y no se presentan hemorragias post-alumbramiento.

La dosis de Tolserol más eficaz fue de 5 gramos en solución dextrosada al $5 \%$. De 60 a 70 gotas por minuto por via endovenosa.

Está indicado el Tolserol en la conducción del parto, en las distocias del cuello uterino por espasmos, en pacientes excitadas durante el trabajo del parto, y $\cong n$ las primíparas añosas.

Estas observaciones se hicieron en número de 13 pacientes. Presentamos como ejemplo las siguientes historias:

Paciente de 35 años, primípara con un embarazo a término, estado general de la enferma satisfactorio. A las 45 horas de comenzado el trabajo del parto 5 
con contracciones uterinas irregulares de mala intensidad y corta duración y presentado síndrome de Schikelée se le aplican 3 gramos de Tolserol por vía endovenosa gota a gota en un tiempo de 50 minutos. A los 10 minutos de aplicada la venoclisis la paciente presenta excitación, euforia y movimientos incoordinados de las manos, se presenta un ligero estado de adormecimiento. La dilatación del cuello uterino al comienzo de la aplicación del Tolserol era de 2 centímetros, a los 65 minutos estaba completa.

Paciente de 21 años, primípara, con un embarazo a término, estado general de la paciente satisfactorio. Ha trabajado 32 horas antes de la aplicación del Tolserol con contracciones uterinas irregulares de corta duración y buena intensidad. Al comienzo de la invección la dilatación del cuello uterino era de 2 centímetros, después de 45 minutos la dilatación estaba completa. Se emplearon 6 gramos de Tolserol vía endovenosa gota a gota. Durante el trabajo presentó somnolencia, relajación muscular y analgesia.

\section{B I B I O G R A F I A}

1.-Berger F. M. and Bradley W.: "The pharmacologic properties of Myanesin" Brit J. Pharmacol. I: 265-272. (Dec. 1946).

2.-Berger F. M. and Bradley W.: "Muscle-relaxin action of Myanesin". - Lancet 252: 97. ( $\operatorname{Jan} 18,1947)$.

3.-Berger F. M.: "The mode of action of Myanesin". - Brit. J. Pharmacol. 2: 241-250. (Dec. 1947).

5.-Pugh J. I. and Enderby G. E. Hale: "Haemoglobinuria after intravenous Myanesin". Lancet 253: 387-388. (Sept. 13, 1947).

6.-Brook, D. M., Hanfield-Jones, Elizabeth: "Effects of Myanesin on muscles and Blood". Lancet 254: 306-307. (Feb. 21, 1948).

7.-Torrens, J. M.; Edward, P. M. and Wood M. W.: "Myanesin in Tetanus". Lancet 255: 807-809. (Nov. 20, 1948).

8.-Hunter, A. R. and Waterfall, Joan W.: "Myanesin in hiperkinetic states". Lancet 254: 366-367. (Mar. 6, 1948).

9.-Noble, C. B.: "Myanesin anesthesia". - Lancet 254: 192. (Jan 31, 1948).

10. -Cowen, J.: "Toxin effects of Myanesin". - Lancet 254: 965-966 (June 19, 1948).

11.-Ogilvie, T. A., Penfold, J. B. and Clendon, D. R. T.: "Gangrene ferrlowing intra-arterial injection of Myanesin with a study of blood an Myanesin mixtures". - Lancet 254: 947-949. (June 19, 1948).

12.-Dexeus Font: "Tratado de Obstetricia". - I: 222-237. (Primera Edición 1949).

13.-Pérez, Manuel Luis: "Tratado de Obstetricia". - 107-110. (Febrero de 1949). 
14. -Smith, Richard T.: "The treatment of some acute rheumatic disorders". - MI. Clin. North America 33: 1.619-1.627. (Nov. 1949).

15.-Gammon, G. D. and Churchill, J. A.: "Effects of Myanesin upon the central nervous system". Am. J. A. Sc. 217: 143-148. (Feb. 1949).

16. -Uriberal, Enrique y Jordán, Jorge: "Drogas modernas en el tratamiento del parkinson y enfermedades similares". - Rev. Médica de Chile 78: 451-456. (Julio de 1949).

17. -Davison, M. H. Armstrong: "Haemolutic effects of Myanesin". - Lancet 257. 1.110. (Dec. 10, 1949).

18.-Clendon, D. R. T. and Penfold, J. B.: "Haemolutic effects of Myanesin". - Lancet 257: 987-989. (Nov. 26, 1949).

19. - Henneman, Elwood and Scherrer, Jean: "The mode of action of Myanesin on the central nervous system". - Tr. Am. Neurol A. 74: 208-211 (1949).

20.-Botella, José: “Fisiología Obstétrica”. (1949).

21.-Kaada, Birger R.: "Site of action of Myanesin in the nervous system". - J. Neurophysiol 13: 98-104. (Jan 1950).

22.-Stumbo, Ellis: "Mephenesin in psychiatric conditions". - Lancet 259: 931-932. (Dec. 30, 1950).

23. - Gottesfeld, B. H.; Mann, Norman and Conway, Edward: "The role of the internist in the treatment of alcoholism". - Connecticut M. J. 15: 678-681. (Aug. 1951).

N. de la R. - Nos parece peligrosa la droga. Tiene graves inconvenientes. Dificil su manejo. 